\title{
External Validation and Sub-cohort Analysis of Stochastic Forecasting Models in NICU Cohorts
}

\author{
Jennifer L. Dickson*, Richard P Floyd*, Aaron J. Le Compte*, Liam M. Fisk*, J. Geoffrey Chase*, \\ Adrienne Lynn**, Geoffrey M. Shaw*** \\ * Department of Mechanical Engineering, University of Canterbury, Christchurch, New \\ Zealand \\ ** Neonatal Department, Christchurch Women's Hospital, Christchurch, New Zealand \\ *** Department of Intensive Care, Christchurch Hospital, Christchurch, New Zealand
}

Abstract: Hyperglycaemia is a prevalent complication in the neonatal intensive care unit (NICU) and is associated with worsened outcomes. It occurs as a result of prematurity, under developed endogenous glucose regulatory systems and clinical stress. The stochastic targeting (STAR) framework provides patient-specific, model-based glycaemic control with a clinically proven level of confidence on the outcome of treatment interventions, thus directly managing the risk of hypo- and hyperglycaemia. However, stochastic models that are over conservative can limit control performance. Retrospective clinical data from 61 episodes (25 retrospective and 36 from a prospective blood glucose control study) of insulin therapy in very-low birth weight (VLBW) and extremely-low birth weight (ELBW) neonates are used to create a new stochastic model of model-based insulin sensitivity $\left(S_{I}[\mathrm{~L} / \mathrm{mU} / \mathrm{min}]\right)$. Sub-cohort models based on gestational age (GA) and birth weight (BW) are also created. Performance is assessed by the percentage of patients who have $90 \%$ of actual intra-patient variability in $S_{I}$ captured by the $90 \%$ confidence bands of the cohort based (inter-patient) stochastic variability model created. This assessment measures per-patient accuracy for any given cohort model.

Per-patient coverage trends were very similar between prospective and retrospective cohorts, providing a measure of external validation of cohort similarity. Per-patient coverage was improved though the use of BW and GA dependent stochastic models, which ensures that the stochastic models more accurately capture both inter- and intra- patient variability. Stochastic models based on insulin sensitivities during insulin treatment periods are tighter and give better and safer glycaemic control. More patient specific methods, particularly in the modeling of endogenous insulin and glucose production, will be required to further improve forecasting and glycaemic control.

Keywords: Insulin sensitivity, control algorithms, physiological models, simulation, intensive care 


\subsection{INTRODUCTION}

Premature infants are a large proportion of neonatal intensive care unit (NICU) populations. Severity of prematurity is commonly quantified by gestational age (GA) and birth weight (BW). Birth weight classifies infants into low birth weight ( $\mathrm{LBW}<2,500 \mathrm{~g}$ ), very low birth weight (VLBW $<1,500 \mathrm{~g}$ ) and extremely low birth weight (ELBW $<1,000 \mathrm{~g}$ ). Similarly GA classifies prematurity as preterm $(<36$ weeks), very preterm $(<31$ weeks $)$ and extremely preterm $(<27$ weeks). Each classification carries increased risk of long term complications, impaired development, and mortality with decreasing BW and GA.

Persistent hyperglycaemia is reported in 57\% of ELBW infants (Hays et al., 2006), and in a study of VLBW infants $80 \%$ had blood glucose $(\mathrm{BG})>8 \mathrm{mmol} / \mathrm{L}, 57 \%$ had $\mathrm{BG}>10 \mathrm{mmol} / \mathrm{L}$, and $32 \%$ had $\mathrm{BG}>10 \mathrm{mmol} / \mathrm{L}$ for more than $10 \%$ of the time (Beardsall et al., 2010). Hyperglycaemia is typically regarded as $\mathrm{BG}$ greater than $10 \mathrm{mmol} / \mathrm{L}$, but there is no standard definition, nor an accepted threshold for intervention (Alsweiler et al., 2007). Hyperglycaemia has been linked to worsened outcome, but no study has conclusively determined if hyperglycaemia itself is harmful, or simply represents severity of condition. The associated negative outcomes include sepsis, increased ventilator dependence, retinopathy of prematurity, increased hospital length of stay, and mortality (Alaedeen et al., 2006; Heimann et al., 2007).

The approach to managing hyperglycaemia is different for each NICU, but effective treatment remains elusive. Current treatments include glucose restriction, and insulin. However, glucose restriction (Hemachandra et al., 1999) deprives the neonate of energy vital for growth and development (Cowett et al., 2004), and is therefore not ideal. The use of insulin infusions to treat hyperglycaemia and/or promote growth has shown positive outcomes including reduced proteolysis, improved glucose tolerance, increased insulin-like 
growth factor (IGF-I) levels, and improved caloric intake and weight gain (Agus et al., 2004; Beardsall, Ogilvy-Stuart, et al., 2007; Beardsall et al., 2008).

However, many insulin trials were unsuccessful in safely providing glycaemic control due to increased hypoglycaemia (Beardsall et al., 2008; Meetze et al., 1998). All reported insulin therapy trials used protocols that fixed insulin dosing based on weight or other factors (Beardsall, Vanhaesebrouck, et al., 2007) or depended on clinical judgement to determine insulin infusion rates. Implicitly, these protocols assume a fixed insulin sensitivity, and so these protocols fail to account for the large intra- and inter-patient variability observed in the insulin sensitivity of neonates (Le Compte et al., 2010; Le Compte et al., 2012). Increased variability with fixed or relatively fixed insulin dosing protocols results in poor control, excessive glycaemic variability and hypoglycaemia (Chase et al., 2011).

STAR (Stochastic TARgeting) is a model based glycaemic control framework (Le Compte et al., 2009; Le Compte et al., 2012) for insulin therapy that uses a time varying insulin sensitivity $\left(S_{I}[\mathrm{~L} / \mathrm{mU} / \mathrm{min}]\right)$ to provide an adaptive patient-specfic response that allows for both inter-patient variability and future intra-patient variability over time. This insulin sensitivity characterises a patient's current metabolic state, and likely future changes in that state are forecast using population based stochastic modelling. A range of possible future insulin sensitivity outcomes is generated based on a patient's current insulin sensitivity, and this enables a treatment to be selected that best overlaps the range of possible BG outcomes with a clinically defined target band. The algorithm for STAR is shown in the appendix. STAR has been the clinical standard of care in Christchurch Women's Hospital since 2009. 
The performance of STAR is dependent on the effectiveness of stochastic modelling; poor stochastic forecasting results in poor glycaemic control. High variability in insulin sensitivity over time and between patients has been shown to limit possible performance of glycaemic control in simulation (Dickson et al.). High variability results in overly conservative stochastic models for some neonates, with wide stochastic forecasting bands that are not representative of all neonates resulting in low doses of insulin and persistently high BG. To enable better and equally safe control for all patients, the stochastic models need to be improved. One avenue is to create stochastic models for the variation of $S_{I}$ over specific subcohorts by GA and BW, which are variables readily available at bedside. The goal is to create models that not only account for inter-patient variability over cohorts, but also capture intrapatient variability (per-patient) more accurately. This is done through a retrospective analysis of insulin sensitivities from clinical data. Quantification of variability and its sources will enable tighter glycaemic control without sacrificing safety. 


\subsection{METHODS}

\subsection{System model}

The clinically validated NICING model (Le Compte et al., 2009) describes glucose-insulin dynamics in the extremely preterm neonate. The model is described by the ordinary differential equations (ODEs) shown in Equations 1-7. Model parameters and variables are defined in Table 1, and the model is shown pictorially in Figure 1.

The rate of change of plasma glucose $(\dot{G})$ is defined in Equation. 1:

$$
\dot{G}=-p_{G} G(t)-S_{I} G(t) \frac{Q(t)}{1+\alpha_{G} Q(t)}+\frac{P(t)+E G P * m_{\text {body }}-C N S * m_{\text {brain }}}{V_{\text {g,frac }}(t) * m_{\text {body }}}
$$

Insulin-mediated glucose clearance is determined by insulin sensitivity $\left(S_{I}\right)$ and non insulinmediated uptake includes a general clearance term, including kidney clearance, $\left(p_{G}\right)$ and a central nervous system uptake (CNS). Glucose sources include exogenous glucose $(P(t))$ and endogenous production $(E G P)$.

The rate of change of plasma $(\dot{I})$ and interstitial $(\dot{Q})$ insulin are defined in Equations 2-4:

$$
\begin{gathered}
\dot{I}=-\frac{n_{L} I(t)}{1+\alpha_{I} I(t)}-n_{K} I(t)-n_{I}(I(t)-Q(t))+\frac{u_{e x}(t)}{V_{I, \text { frac }} * m_{\text {body }}}+\left(1-x_{L}\right) u_{e n} \\
u_{e n}=\mathrm{I}_{\mathrm{B}} e^{\frac{-k_{l} u_{e x}}{V_{I}}} \\
\dot{Q}=n_{I}(I(t)-Q(t))-n_{C} \frac{Q(t)}{1+\alpha_{G} Q(t)}
\end{gathered}
$$

Plasma insulin is cleared via the liver $\left(n_{L}\right)$, the kidney $\left(n_{K}\right)$ and transport into interstitial fluid $\left(n_{I}\right)$. Insulin enters the system exogenously $\left(u_{e x}\right)$ or endogenously $\left(u_{e n}\right)$ through pancreatic secretion, as described in Eqn. 3. Insulin leaves the interstitial fluid through degradation $\left(n_{C}\right)$. Appearance of glucose via the enteral route is modelled via two intermediary compartments, the stomach $\left(P_{1}\right)$ and the gut $\left(P_{2}\right)$, and is described in Equations 5-7.

$$
\begin{gathered}
\dot{P}_{1}=-d_{1} P_{1}+P(t) \\
\dot{P}_{2}=-\min \left(d_{2} P_{2}, P_{\max }\right)+d_{1} P_{1}
\end{gathered}
$$




$$
P(t)=\min \left(d_{2} P_{2}, P_{\max }\right)+P N(t)
$$

Transport rates between the stomach and gut, and gut and blood ( $d_{1}$ and $d_{2}$ respectively) are limited to a maximum flux $\left(P_{\max }\right)$. Solutions to equations 1-7 (giving profiles for G,I,Q, $\mathrm{P}_{1}$ and $\mathrm{P}_{2}$ ) are generated simultaneously in the time domain using a Runga-Kutta 4 based ODE solver.

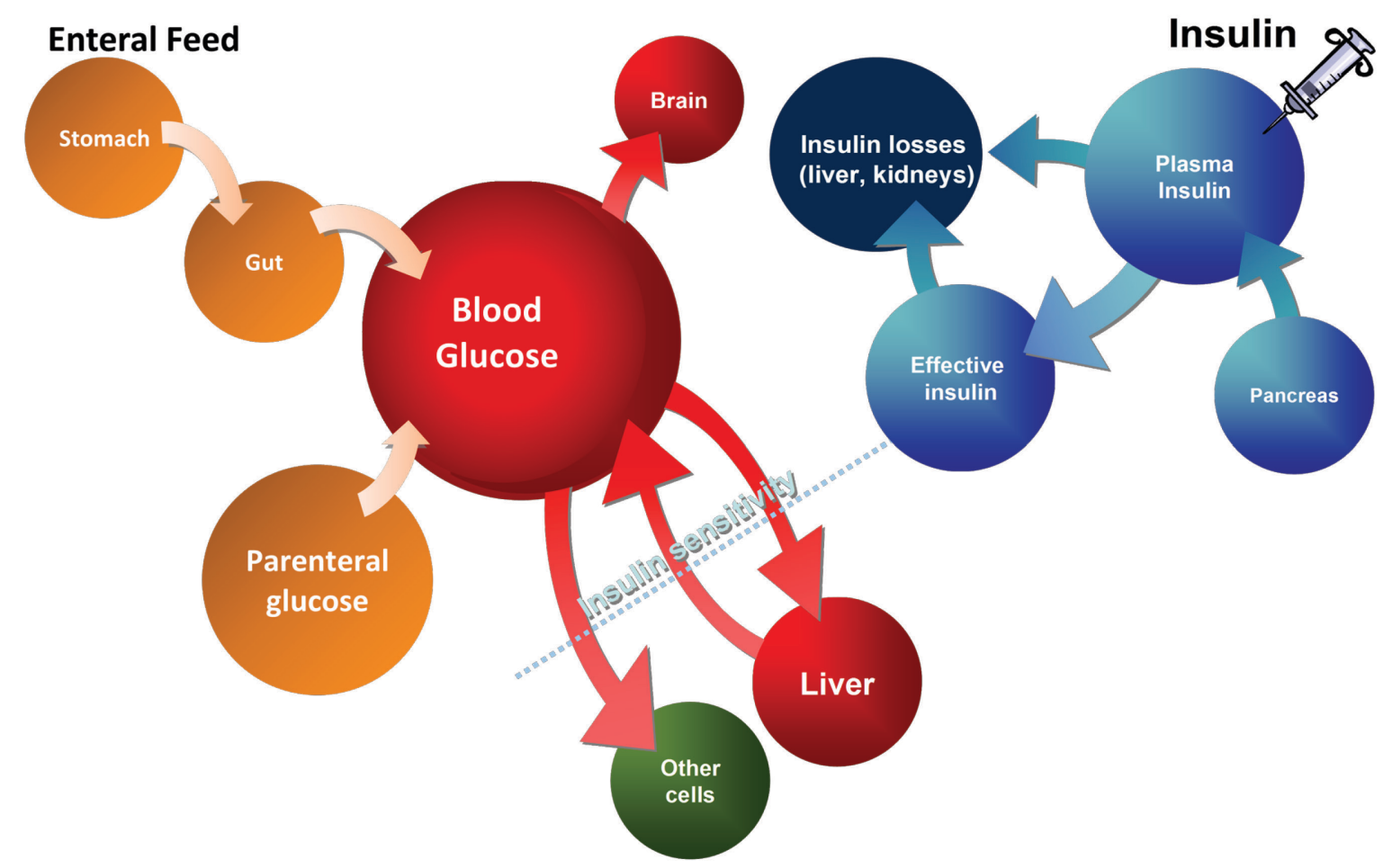

Figure 1: Pictorial representation of the NICING model.

$S_{I}$ is patient specific and time varying, describing a patients current metabolic state. It is fit using integral based fitting methods (Hann et al., 2006) on a retrospective hour-to-hour basis, and assumed constant over an hour-long period. In addition to being a marker of peripheral insulin sensitivity, $S_{I}$ also incorporates uncertainty around patient-specific endogenous insulin and glucose production. A $S_{I}$ of zero represents the lower physiological bound in insulin sensitivity where no glucose is leaving the blood plasma via the insulin-mediated uptake path. 
Table 1: Glucose-insulin metabolic model variable definition

\begin{tabular}{|c|c|c|}
\hline Variable & Description & Values \\
\hline$G$ & Blood glucose level & $(\mathrm{mmol} / \mathrm{L})$ \\
\hline$I$ & Plasma insulin concentration & $(\mathrm{mU} / \mathrm{L})$ \\
\hline$Q$ & Interstitial insulin concentration & $(\mathrm{mU} / \mathrm{L})$ \\
\hline$p_{G}$ & Endogenous glucose clearance & $0.0030\left(\mathrm{~min}^{-1}\right)$ \\
\hline$\alpha_{G}$ & Saturation parameter for insulin mediated glucose removal & $0(\mathrm{~L} / \mathrm{mU})$ \\
\hline$\alpha_{I}$ & Saturation parameter for plasma insulin clearance & $0.0017(\mathrm{~L} / \mathrm{mU})$ \\
\hline$S_{I}$ & Insulin sensitivity & $(\mathrm{L} / \mathrm{mU} / \mathrm{min})$ \\
\hline$E G P$ & Endogenous glucose production & $\begin{array}{c}0.0284 \\
(\mathrm{mmol} / \mathrm{kg} / \mathrm{min})\end{array}$ \\
\hline CNS & Central nervous system glucose uptake & $0.088(\mathrm{mmol} / \mathrm{kg} / \mathrm{min})$ \\
\hline$P(t)$ & Glucose appearance in plasma from dextrose intake & $(\mathrm{mmol} / \mathrm{min})$ \\
\hline$P N$ & Parenteral Nutrition & $(\mathrm{mmol} / \mathrm{min})$ \\
\hline$P_{\max }$ & Maximal glucose flux from gut to plasma & $6.11(\mathrm{mmol} / \mathrm{min})$ \\
\hline$P 1$ & Glucose level in stomach & $(\mathrm{mmol})$ \\
\hline$P 2$ & Glucose level in gut & $(\mathrm{mmol})$ \\
\hline$V_{G}$ & Plasma glucose distribution volume & $0.5961(\mathrm{~L})$ \\
\hline$k_{I}$ & Interstitial insulin transport rate & $0.1\left(\min ^{-1}\right)$ \\
\hline$I_{B}$ & Endogenous insulin production & $15(\mathrm{mU} / \mathrm{L} / \mathrm{min})$ \\
\hline$n_{I}$ & $\begin{array}{l}\text { Rate of transport between plasma and interstitial insulin } \\
\text { compartments }\end{array}$ & $0.003\left(\mathrm{~min}^{-1}\right)$ \\
\hline$n_{K}$ & Renal insulin clearance & $0.150\left(\mathrm{~min}^{-1}\right)$ \\
\hline$n_{L}$ & Hepatic insulin clearance & $1\left(\min ^{-1}\right)$ \\
\hline$n_{C}$ & Interstitial insulin degradation & $0.003\left(\mathrm{~min}^{-1}\right)$ \\
\hline$x_{L}$ & First-pass hepatic insulin clearance & 0.67 \\
\hline$u_{e x}(t)$ & Exogenous insulin & $(\mathrm{mU} / \mathrm{min})$ \\
\hline$u_{e n}(t)$ & Endogenous insulin production & $(\mathrm{mU} / \mathrm{L} / \mathrm{min})$ \\
\hline$V_{I}$ & Plasma insulin distribution volume & $0.0450(\mathrm{~L})$ \\
\hline$d_{1}$ & Glucose absorption rate from stomach & $0.0347\left(\mathrm{~min}^{-1}\right)$ \\
\hline$d_{2}$ & Glucose absorption rate from gut & $0.0069\left(\mathrm{~min}^{-1}\right)$ \\
\hline$D(t)$ & Dextrose intake & $(\mathrm{mmol} / \mathrm{min})$ \\
\hline$m_{\text {body }}$ & Body mass & $(\mathrm{kg})$ \\
\hline$m_{\text {brain }}$ & Brain mass $\left(14 \% \boldsymbol{m}_{\text {body }}\right)$ & $(\mathrm{kg})$ \\
\hline
\end{tabular}




\subsection{Clinical Patients and Insulin Sensitivity Fitting}

$S_{I}$ profiles were fit using integral based fitting (Hann et al., 2006) from clinical data using Equations 1-7. This clinically validated metric and method (Chase et al., 2010) allows the performance and stochastic forecasting of STAR to be optimised before clinical trials. The integral based fitting method can be used since $S_{I}$ is constant across an hour long time interval. If this time interval is bounded by $t_{1}$ and $t_{2}$, SI can be solved for by the re-arranging of the integrated form of Equation 1 for $\mathrm{S}_{\mathrm{I}}$, giving Equation 8.

$$
S_{I}=\frac{-\left(G\left(t_{2}\right)-G\left(t_{1}\right)\right)+\int_{t_{1}}^{t_{2}}\left\{-p_{G} G(t)+\frac{P(t)+E G P * m_{\text {body }}-C N S * m_{\text {brain }}}{V_{\text {g,frac }}(t) * m_{\text {body }}}\right\} d t}{\int_{t_{1}}^{t_{2}}\left\{\frac{Q(t)}{1+\alpha_{G} Q(t)}\right\} d t}
$$

The start and end BG for each hour of clinical data, $G\left(t_{2}\right)$ and $G\left(t_{1}\right)$, is approximated using linear interpolation between BG measures. The $S_{I}$ profile of a patient consists of a constant $S_{I}$ identified for each hour of clinical data.

The patient cohort, summarised in Table 2, consists of data from 21 retrospective patients (with 25 patient episodes), and 8 short term and 22 long term patients from a prospective BG control study using STAR. The 8 short term patients received insulin therapy for 24 hours in a validation trial of the existing model and controller (Le Compte et al., 2009). Long term patients were treated using STAR as a standard of care at Christchurch Women's Hospital. There are 61 clinical patient datasets, as there are 28 treatment episodes for the 22 long term patients.

Table 2: Clinical patient summary statistics.

\begin{tabular}{|c|c|c|c|c|c|c|c|c|c|c|c|}
\hline & \multicolumn{4}{|c|}{ Short-term $(\mathrm{N}=8)$} & \multicolumn{4}{|c|}{ Long-term $(\mathrm{N}=28)$} & \multicolumn{3}{|c|}{ Retrospective $(\mathrm{N}=25)$} \\
\hline & Median & [IQR] & & & Median & [IQR] & & & Median & [IQR] & \\
\hline $\begin{array}{l}\text { Gestational age at birth } \\
\text { (weeks) }\end{array}$ & 25.6 & {$[24.9$} & - & 26.4] & {$[25.4$} & {$[25.0$} & - & 26.8] & 26.6 & {$[25.4$} & $\begin{array}{ll}- & 27.7\end{array}$ \\
\hline $\begin{array}{l}\text { Weight at birth } \\
\text { (grams) }\end{array}$ & 745 & {$[681$} & - & 814] & 760 & {$[601$} & - & 925] & 845 & {$[800$} & $\begin{array}{ll}- & 904]\end{array}$ \\
\hline $\begin{array}{l}\text { Age at start of trial } \\
\text { (days) }\end{array}$ & 6.6 & {$[3.6$} & - & 7.7] & 3.6 & {$[1.5$} & - & $6.4]$ & $\mathrm{n} / \mathrm{a}$ & & \\
\hline
\end{tabular}




\subsection{Improving Stochastic Forecasting with Increased Cohort}

The current stochastic model used in the STAR controller was designed using a retrospective cohort of 25 patients (Le Compte et al., 2010). Hence, the relevance of its performance in virtual trials may be limited. A stochastic matrix created using the larger 61 virtual patient cohort is compared to the current stochastic matrix.

The stochastic model used for $S_{I}$ forecasting is generated from changes in insulin sensitivity across a retrospective representative clinical patient population. Changes in $S_{I}$ can be seen when a $S_{I}$ value $\left(S_{I, n}\right)$ is plotted against the $S_{I}$ value 1-4 hours forward $\left(S_{I, n+1}\right)$, as shown in Figure 2. No change would result in a straight line with gradient 1 . Kernel density methods are used to generate a distribution of likely future values of $S_{I}$ for each sensitivity for which an example is shown in Figure 2. Forecasting is achieved using the $5^{\text {th }}$ and $95^{\text {th }}$ percentiles of likely $S_{I}$ outcome.

Performance of retrospective and prospective clinical sub-cohort based stochastic models is compared with a whole cohort model over 3-hour forecast intervals. A perfect stochastic model would capture $90 \%$ of each individual patient's variations in the $5-95^{\text {th }}$ percentile interval, but this may vary for individual patients. Performance of each stochastic model is assessed by the percentage of patients whose individual stochastic performance comes close to this ideal, for a more accurate and general the stochastic model. 


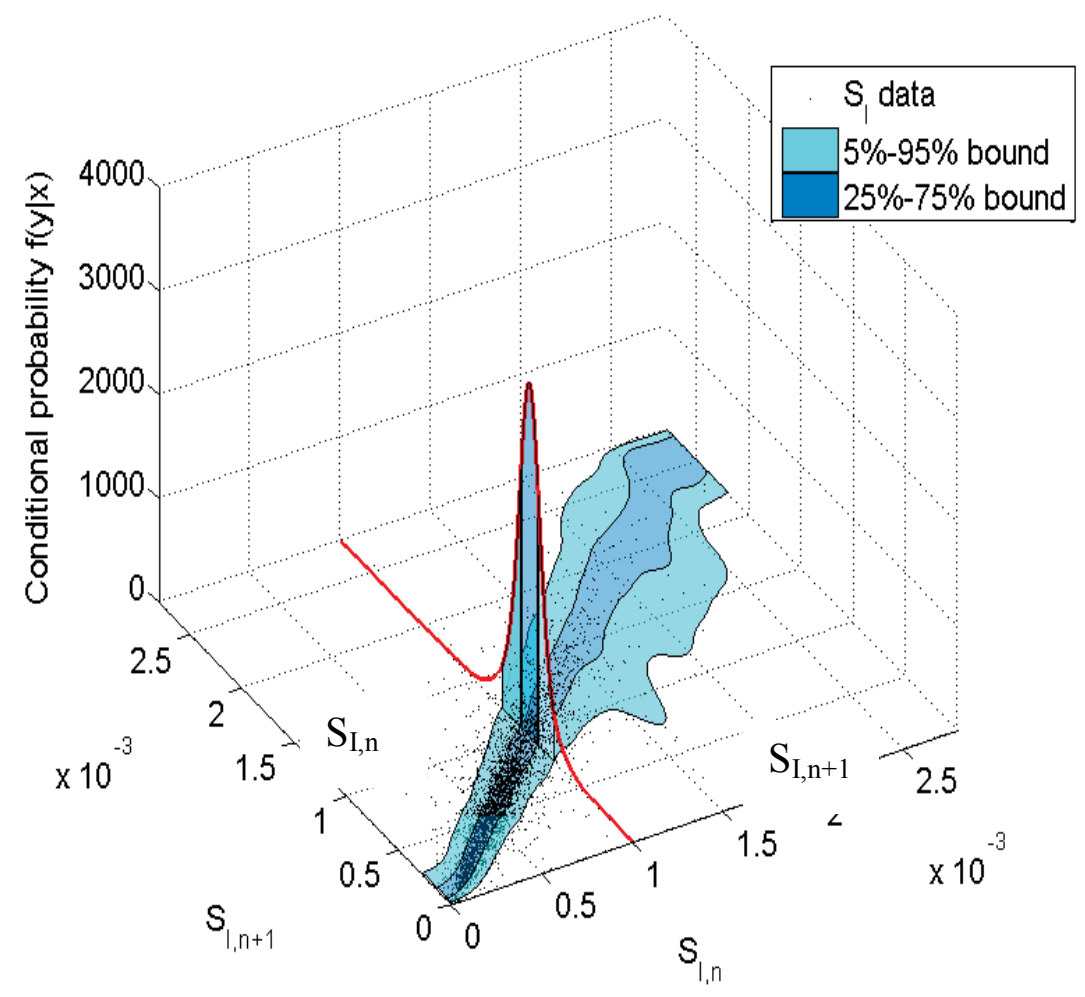

Figure 2: Hourly insulin sensitivity variation data with probability bounds and example curve showing probability bounds (Le Compte et al., 2010). 


\subsection{Improving Stochastic Forecasting in Sub-Cohorts}

Two variables easily identified at the bedside are birth weight (BW) and gestational age (GA). Stochastic model matrices are created for each sub-cohort generated by tertiles in BW or GA. A Kolmogorov-Smirnov (KS) test was used to identify the most significantly different patient sub-cohort groupings by absolute $S_{I}$ and hour-to-hour changes in $S_{I}$. Subcohort groupings were arranged by $\mathrm{BW}$ or GA. BW based sub-cohorts were arranged in several ways: 1) by tertiles and 2) into two groups with the critical BW ranging from 700 to $1300 \mathrm{~g}$ in 5 gram steps. Similarly GA sub-cohorts were arranged by: 1) tertiles, and 2) two groups with the critical GA ranging from 24 to 25 weeks. From the patient sub-cohort groupings that were most different $(\mathrm{p}<0.005)$ additional stochastic models were created to differentiate behaviours in $S_{I}$. Performance is assessed using per-patient coverage.

\subsection{Improving Stochastic Forecasting using only Insulin Sensitivities from Insulin therapy} periods

Inherent in the clinical data are periods of several hours in length where the patient has not received at exogenous insulin. These periods tend to occur at the conclusion of a patient episode and reflect the improving ability of a patient to regulate their own plasma glucose levels. Stochastic models were developed using only the patient data in which exogenous insulin had been given sometime within the last two hours. Performance is assessed using per-patient coverage, and is directly compared to the non-insulin specific case.

\subsection{Clinically validated virtual trial methods}


The effect of stochastic modelling on the tightness and performance of glycaemic control can be evaluated through clinically validated virtual trial methods (Chase et al., 2010). Virtual trials test the effect of insulin and nutrition treatments on a virtual patient's blood glucose by using a known, treatment independent (Chase et al., 2010), insulin sensitivity profile derived from clinical data. Future information is ignored and the simulation algorithm in Appendix 2 is followed. The change in blood glucose levels over the intervention interval is then simulated using the known insulin sensitivity profile. Control performance outcomes are measured in the percentage time in the $4-8 \mathrm{mmol} / \mathrm{L}$ band, percentage $\mathrm{BG}>10 \mathrm{mmol} / \mathrm{L}$, and safety is evaluated in the percentage $\mathrm{BG}<4.0 \mathrm{mmol} / \mathrm{L}$ and number of incidences of $\mathrm{BG}<2.6$ $\mathrm{mmol} / \mathrm{L}$. 


\subsection{RESULTS}

\subsection{Stochastic Forecasting with Increased Cohort}

Figure 3 shows the per-patient coverage of the $5^{\text {th }}$ to $95^{\text {th }}$ percentile of forecasted change in $S_{I}$ for the current $(\mathrm{N}=25)$ and new $(\mathrm{N}=61)$ whole-cohort stochastic models. There is no significant difference between the per-patient coverage of the different stochastic matrices. Both stochastic matrices have a minimum coverage over $70 \%$ and tight distributions around $90 \%$. This result provides a measure of external validation in that the retrospective $(\mathrm{N}=25)$ cohort showed similar behaviour and performance to the prospective $(\mathrm{N}=36)$ cohort. The same results can be seen for 2 and 4 hour measurement intervals (not shown). However, small improvements can be seen in the coverage distribution for the new whole-cohort stochastic matrix ( $\mathrm{N}=61)$. It is important to note that Figure 3 is the per-patient coverage, and not all patients have equal numbers of measurements and thus, do not have the same weighting on stochastic model forecast limits. In addition, manipulation of stochastic matrices to bring the overall whole-cohort percentage coverage closer to the target $90 \%$ tends to adjust the percentiles to capture a single data point, thus making the matrix more cohortspecific and adding no extra value for use outside of the existing cohort.

\subsection{Gestational Age and Birth Weight Sub-Cohort}

The cumulative distribution functions (CDFs) of $S_{I}$ for tertiles of is shown in Figures 3. The CDFs of SI when separated by GA ( $<25.1$ weeks, $>26.9$ weeks, and between 25.1 and 26.9 weeks) are almost identical to Figure 4 . The lower tertile has significantly lower $S_{I}$ than the other groups for both GA and BW ( $\mathrm{p}<0.05, \mathrm{KS}$-test). In both cases, the relative changes in $S_{I}$ (lower plot) are not significantly different. Thus, these tertiles are different in absolute $S_{I \text {, }}$ but not in hour-to-hour variability. 

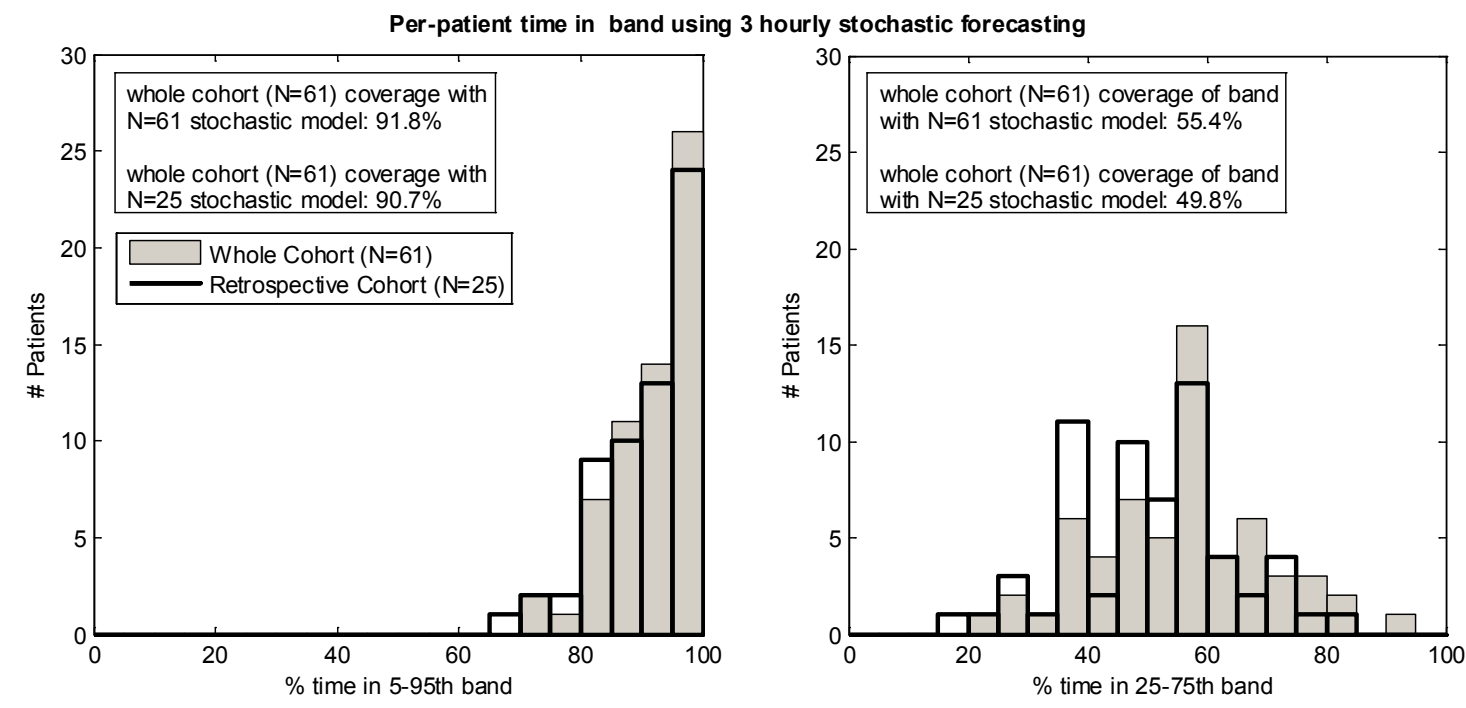

Figure 3: Per-patient coverage between the forecasted $5^{\text {th }}$ and $95^{\text {th }}$, and $25^{\text {th }}$ and $75^{\text {th }}$, percentile change in insulin sensitivity using the current stochastic matrix compared with new stochastic matrix. ( $\mathrm{N}=$ number of patients used to create stochastic matrix).

Table 3 shows the results for the tertiles in Figures 4, as well as other statistically significant cut-off values found $(\mathrm{p}<0.05)$ for $S_{I}$ over BW and GA sub-cohorts. Figure 5 shows the resulting per-patient coverage for stochastic forecasting using stochastic model for each of the weight tertiles, on comparison to a whole cohort stochastic model. There is noticeable improvement from the whole cohort $(\mathrm{N}=61)$ result shown for comparison, with increased patient coverage around $90 \%$. This result was typical for all the stochastic model combinations shown in Table 3. Thus, the BW and GA dependent models add greater resolution and per-patient accuracy. 
Table 3: Effect of weight and gestational age on insulin sensitivity statistics.

\begin{tabular}{|c|c|c|c|c|}
\hline Data Set & $\begin{array}{c}S_{I} \text {-Median [IQR] } \\
(\mathrm{L} / \mathrm{mU} / \mathrm{min})\end{array}$ & $\begin{array}{l}\text { Relative delta } S_{I}- \\
\text { Median (P) [IQR] }\end{array}$ & \# hours & \#Patients \\
\hline Whole Cohort & $0.0017[0.0010-0.0027]$ & $0.004204[-0.0303-0.0351]$ & 6968 & 61 \\
\hline $\mathrm{BW}<700 \mathrm{~g}$ & $0.0013[0.0007-0.0020]$ & $0.004116[-0.0299-0.0339]$ & 3032 & 20 \\
\hline $\mathrm{BW}>865 \mathrm{~g}$ & $0.0020[0.0013-0.0031]$ & $0.006074[-0.0289-0.0376]$ & 1426 & 20 \\
\hline $700 \mathrm{~g}<\mathrm{BW}<865 \mathrm{~g}$ & $0.0021[0.0013-0.0032]$ & $0.003114[-0.0312-0.0360]$ & 2510 & 21 \\
\hline $\mathrm{BW}<\mathbf{8 0 5 g}$ & $0.0015[0.0008-0.0025]$ & $0.003640[-0.0310-0.0344]$ & 4566 & 32 \\
\hline $\mathrm{BW}>925 \mathrm{~g}$ & $0.0024[0.0015-0.0037]$ & $0.008583[-0.0194-0.0353]$ & 748 & 12 \\
\hline $805 \mathrm{~g}<\mathrm{BW}<925 \mathrm{~g}$ & $0.0019[0.0013-0.0030]$ & $0.003678[-0.0312-0.0385]$ & 1654 & 17 \\
\hline $\mathrm{BW}<700 \mathrm{~g}$ & $0.0013[0.0007-0.0020]$ & $0.004116[-0.0299-0.0339]$ & 3032 & 20 \\
\hline $\mathrm{BW}>700 \mathrm{~g}$ & $0.0021[0.0013-0.0032]$ & $0.004254[-0.0305-0.0369]$ & 3907 & 40 \\
\hline $\mathrm{BW}<\mathbf{8 0 0 g}$ & $0.0014[0.0007-0.0021]$ & $0.003866[-0.0289-0.0334]$ & 3519 & 27 \\
\hline $\mathrm{BW}>800 \mathrm{~g}$ & $0.0021[0.0013-0.0033]$ & $0.004446[-0.0315-0.0382]$ & 3449 & 34 \\
\hline GA $<25.1 w k s$ & $0.0013[0.0007-0.0021]$ & $0.004001[-0.0314-0.0343]$ & 3196 & 22 \\
\hline GA>26.9wks & $0.0020[0.0013-0.0032]$ & $0.008319[-0.0215-0.0370]$ & 1200 & 16 \\
\hline $25.1<\mathrm{GA}<26.9 \mathrm{wks}$ & $0.0020[0.0013-0.0031]$ & $0.002614[-0.0316-0.0364]$ & 2572 & 23 \\
\hline GA $<26.15 w k s$ & $0.0015[0.0008-0.0026]$ & $0.003139[-0.0316-0.0340]$ & 4561 & 34 \\
\hline GA $>27.05 w k s$ & $0.0021[0.0014-0.0033]$ & $0.008422[-0.0205-0.0374]$ & 1141 & 14 \\
\hline $26.15 w \mathrm{ks}<\mathrm{GA}<27.05$ wks & $0.0019[0.0013-0.0027]$ & $0.004314[-0.0315-0.0389]$ & 1266 & 13 \\
\hline GA $<26.3$ wks & $0.0015[0.0008-0.0026]$ & $0.003138[-0.0310-0.0340]$ & 4842 & 37 \\
\hline GA>26.3 wks & $0.0020[0.0014-0.0030]$ & $0.007230[-0.0287-0.0385]$ & 2126 & 24 \\
\hline GA $<27.05$ wks & $0.0016[0.0009-0.0026]$ & $0.003364[-0.0316-0.0349]$ & 5827 & 47 \\
\hline GA $>27.05$ wks & $0.0021[0.0014-0.0033]$ & $0.008422[-0.0205-0.0374]$ & 1141 & 14 \\
\hline
\end{tabular}

CDF plot of Insulin Sensitivity $\left(S_{f}\right)$ for Birth Weight Tertiles

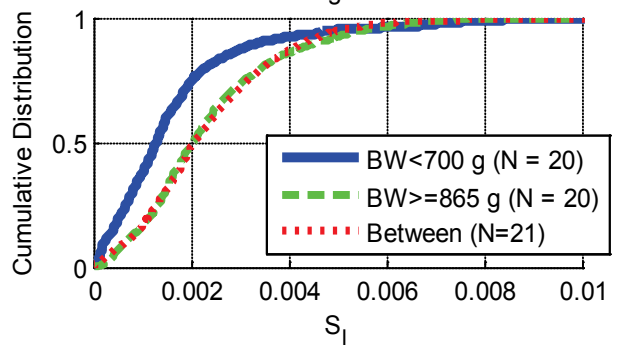

CDF plot of Relative Change in Insulin Sensitivity $\left(S_{p}\right)$ for

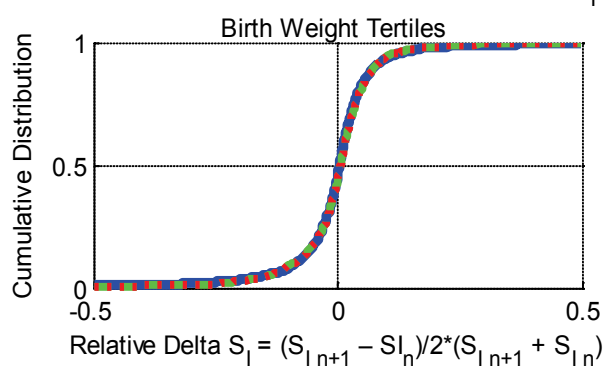

Figure 4: Correlation of insulin sensitivity and change in insulin sensitivity with birth weight

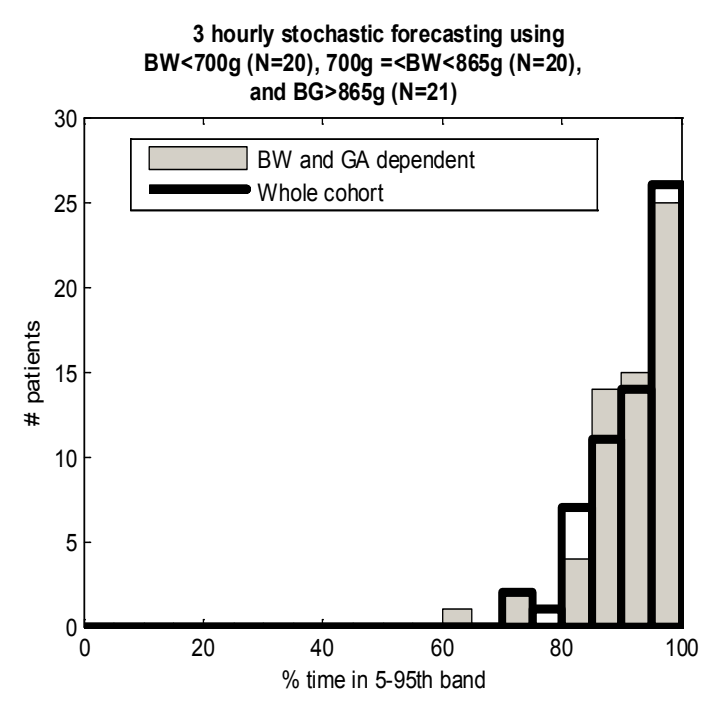

Figure 5: Comparison of per-patient coverage for BW and GA dependent point to point 3 hourly stochastic forecasting. 


\subsection{Low patient coverage case studies}

Given that the whole cohort stochastic models have $\sim 90 \%$ overall coverage in the $5-95^{\text {th }}$ percentile band, patients with individual coverage less than $85 \%$ were extracted for further analysis. As can be seen in Table 4 there is no common characteristic that could potentially be used to identify such patients as more variable. The patients summarised in Table 4 cover the entire range for both gestational age and birth weight. Furthermore, analysis of the perpatient coverage statistics of the entire patient cohort shows no trends with birth weight, gestational age or gender, and while, in general, a patient with low coverage of the $5^{\text {th }}$ to $95^{\text {th }}$ percentile band also had low coverage of the $25^{\text {th }}-75^{\text {th }}$ band (defined as coverage less than $45 \%)$, the same was not true in reverse.

Table 4: Highly variable patient statistics. LT denotes patients from the long term cohort, R denotes patients from the retrospective cohort

\begin{tabular}{|c|c|c|c|c|c|c|c|}
\hline $\begin{array}{l}\text { Patient } \\
\text { Number: }\end{array}$ & $\begin{array}{l}\text { Hours } \\
\text { Hou in }\end{array}$ & $\begin{array}{l}\text { \% above } \\
\mathbf{5}^{\text {th }}-\mathbf{9 5}^{\text {th }} \\
\text { band }\end{array}$ & $\begin{array}{l}\text { \% in } \\
\mathbf{9 5}^{\text {th }} \\
\text { percentile }\end{array}$ & $\begin{array}{l}\mathbf{\%} \text { above } \\
\mathbf{2 5}^{\text {th }}-\mathbf{7 5}^{\text {th }} \\
\text { band }\end{array}$ & $\begin{array}{l}\text { Birth } \\
\mathbf{7 5}^{\text {th }} \\
\text { percentile }\end{array}$ & $\begin{array}{l}\text { Gestational } \\
\text { age }\end{array}$ \\
\hline LT 21 & 272 & 75.1 & 10.0 & 30.9 & 33.5 & 0.605 & 25 \\
\hline LT 27 & 28 & 80.0 & 12.0 & 28.0 & 48.0 & 0.69 & 24.6 \\
\hline R 6 & 93 & 73.3 & 17.8 & 33.3 & 38.8 & 0.900 & 25 \\
\hline R 10 & 66 & 76.2 & 19.0 & 38.9 & 42.8 & 1.28 & 27.9 \\
\hline R 12 & 34 & 80.6 & 19.4 & 29.0 & 35.5 & 0.845 & 28.6 \\
\hline R 19 & 44 & 82.9 & 17.1 & 43.9 & 36.5 & 0.93 & 28.6 \\
\hline
\end{tabular}




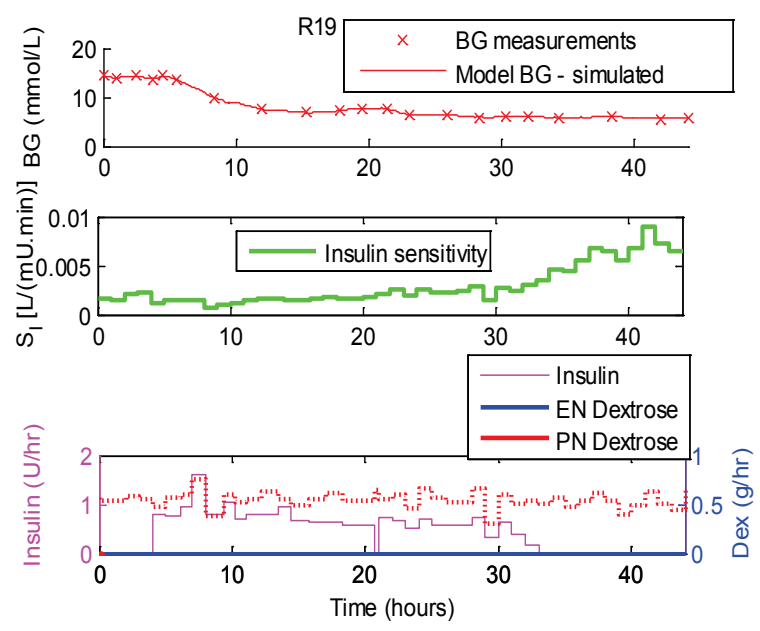

a)

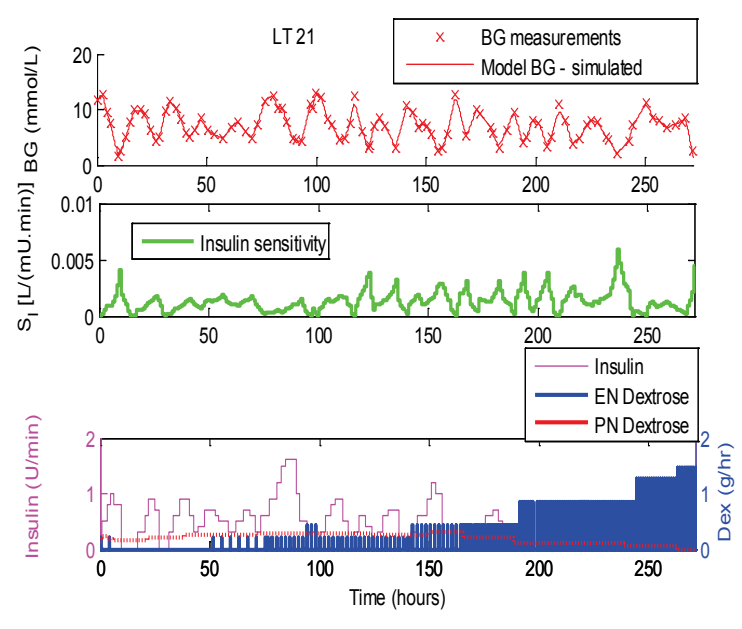

b)

Figure 6: Clincial data and SI profiles for two highly variable patients: a) Retrospective patient 19 and b) Long Term patient 21

Figure 6 shows two high variability profiles derived from clinical data. In Figure 6 a) the $S_{I}$ rises unusually fast and unusually high, whereas in Figure $7 \mathrm{~b}$ ) the $S_{I}$ exhibits sudden peaks and drops. In particular, it is the sudden increases in $S_{I}$ beyond the forecasted bounds that can result in unexpectedly low BG. For this reason, when carrying out virtual trials, the highly variable patients tend to constitute unrepresentative, larger proportions of the low BG $(\mathrm{BG}<4.0 \mathrm{mmol} / \mathrm{L})$ time periods, which means the stochastic matrices remain overly conservative for the remainder of the cohort. The $S_{I}$ of the other high variability patients from Table 4 generally exhibit aspects of both these example profiles, and are difficult to visually identify as differing from the rest of the cohort without looking at per-patient coverage. Patients that are variable with respect to the cohort are thus hard to identify in the clinical situation with the limited bedside metrics available. 


\subsection{Stochastic matrices based on periods of insulin therapy}

The analysis was carried out using $S_{I}$ from time periods in clinical data when a patient had been on insulin within the last two hours. This removed the increase in $S_{I}$ common at the end of a patient's clinical data, when they are not receiving insulin treatments and their BG is basal and stable. This increase in $S_{I}$ reflects an improvement in patient condition. Figure 6 shows the difference made to per patient coverage. The narrowing of stochastic forecasting bands is evident in Figure 7 with the lower overall patient coverage, and the clear distribution around $90 \%$ coverage. There was no difference in the results for trends with BW and GA.

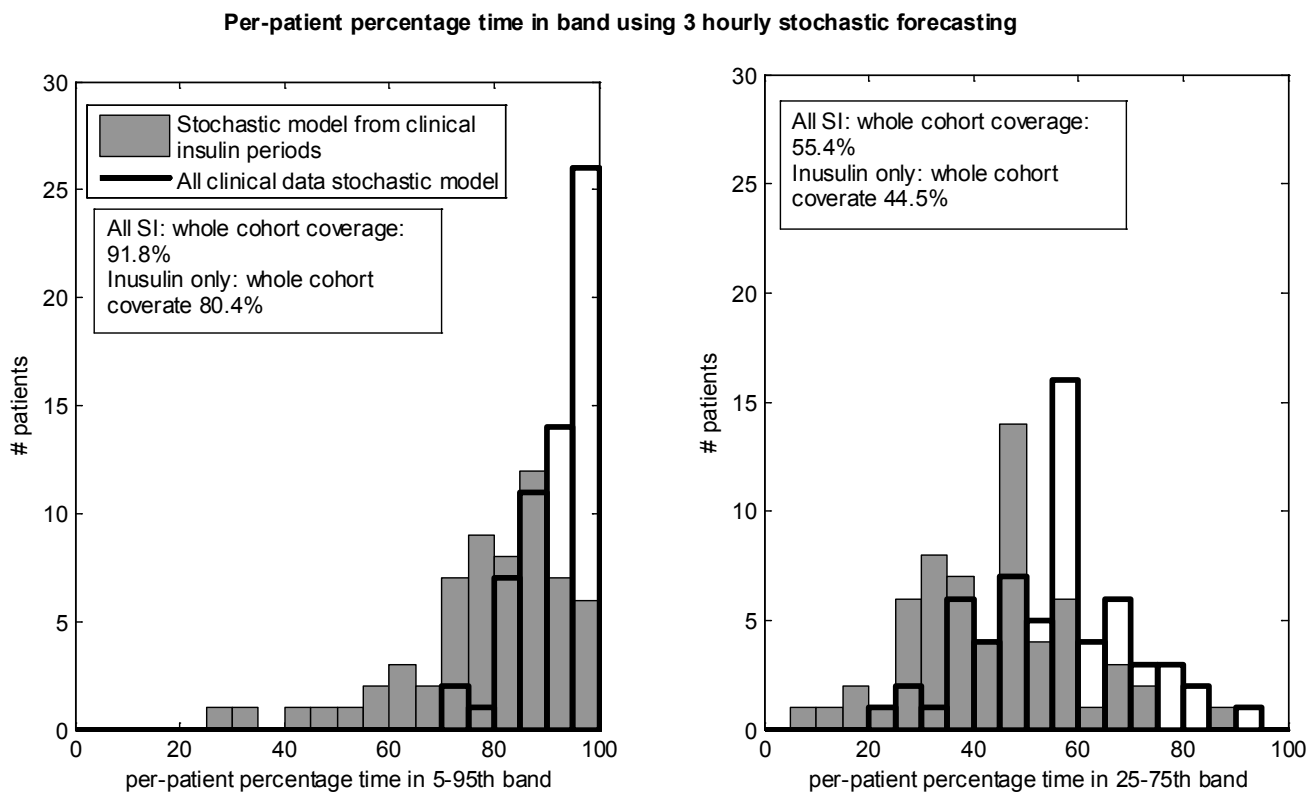

Figure 7: Per-patient coverage between the forecasted $5^{\text {th }}$ and $95^{\text {th }}$ percentile change in insulin sensitivity using the current stochastic matrix compared with an insulin periods only stochastic model. The clinical data used to generate the insulin periods stochastic model ignores any data where insulin has not been given within the last 2 hours.

\subsection{Simulation Results}

Clinically validated virtual trials using the stochastic matrix from insulin periods showed a $10 \%$ increase in performance $(\% \mathrm{BG}$ between 4.0 and $8.0 \mathrm{mmol} / \mathrm{L})$, and a drop in the number 
of hypoglycaemic events $(\mathrm{BG}<2.6 \mathrm{mmol} / \mathrm{L})$ from 4 to 2 patients. This result shows that using insulin-period-based stochastic modelling increases the performance and safety of glycaemic control.

\begin{tabular}{|c|c|c|c|c|}
\hline & 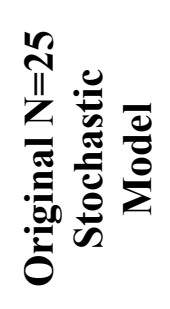 & 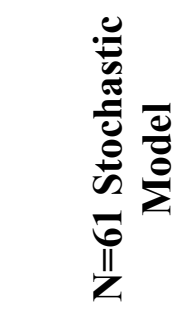 & 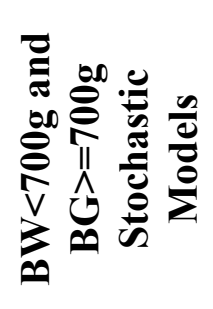 & 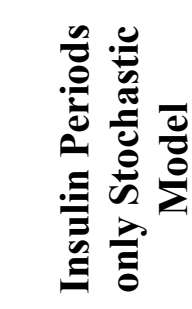 \\
\hline $\begin{array}{l}\text { \% Time in Band } \\
(4.0-8.0 \mathrm{mmol} / \mathrm{L})\end{array}$ & & 64.1 & & 76.6 \\
\hline$\% \mathrm{BG}<4.0 \mathrm{mmol} / \mathrm{L}$ & & 1.7 & & 2.2 \\
\hline $\begin{array}{c}\% \mathrm{BG}<2.6 \mathrm{mmol} / \mathrm{L} \\
\text { (\# patient episodes) }\end{array}$ & & $0.09(3)$ & & $0.1(6)$ \\
\hline$\% \mathrm{BG}>10 \mathrm{mmol} / \mathrm{L}$ & & 14.3 & & 7.6 \\
\hline
\end{tabular}




\subsection{DISCUSSION}

Comparison of per-patient percentile band coverage for different cohort based stochastic matrices has shown that the cohorts are essentially similar in behaviour. The addition of 36 patient episodes $(\mathrm{N}=25$ to $\mathrm{N}=61)$ has improved per-patient stochastic model coverage slightly, but not to an extent that is likely to be clinically significant in use. This similarity in coverage with the use of different cohort stochastic forecasting verifies that the original data set is as representative of the NICU population as previously thought, where initial work with stochastic matrices in adult ICU patients indicated $\mathrm{N}=25$ would be suitable (Lin et al., 2006).

The high proportion of coverage above $90 \%$, and in particular above $95 \%$ in the $5-95^{\text {th }}$ band suggests that this band width is determined by the behaviour of a relative few patients. Ideally, for patient specificity, the majority of per-patient coverage would be close to the target $90 \%$. The patient coverage of the $25-75^{\text {th }}$ percentiles is much wider, indicating significant inter-patient variability and lack of patient-specific coverage within these central tendency bounds. In particular, the short and long term cohorts tend to have higher average coverage in the $25^{\text {th }}-75^{\text {th }}$ band suggesting that differences between cohorts arise within the extremes of behaviour, and that estimates of variability are over conservative in the majority of cases. Overall it seems that inter-patient variation is more significant than intra-patient variation as a limiting factor in this stochastic forecasting model, and that a relative few more variable patients are quite different in behaviour.

BW and GA dependent stochastic models can be used to further improve per-patient coverage, as seen in Figure. The proportion of coverage around the $90 \%$ target for the 5-95 percentiles is much greater, indicating BW and GA can be used to introduce greater patient specificity in stochastic forecasting. Due to the relatively small number of patients used, the ideal combinations of BW and GA found may not fully represent all NICU populations or be 
perfect divisions for other NICU cohorts. Equally, there may be differences between NICUs due to differences in cohort or case mix. However, the results clearly illustrate potential to improve patient-specific forecasting and glycaemic control based on easily measured variables. Further investigations using larger independent cohorts should be completed to validate these initial insights, and create more generalisable results. However, these results provide a template for further analysis.

A small proportion of patients from this cohort exhibit changes in $S_{I}$ that is not well predicted by the whole cohort and birth weight divisional stochastic models. These patients do not have any readily identifiable patient descriptor, such as birth weight or gestational age, in common. However, these patients can be roughly divided according to their exogenous insulin requirements. Several patients had high insulin sensitivity and did not require exogenous insulin to maintain constant $\mathrm{BG}$. Their $\mathrm{S}_{\mathrm{I}}$ was not well predicted by the model due to improvement of patient condition, exacerbating per-patient variability and deviance from modelled insulin and glucose secretion, and thus they are in no danger as high insulin sensitivity will result in no treatment. These cases highlight model specificity for describing glucose and insulin dynamics in the neonate with deficient glycaemic control.

The other type of patient with $\mathrm{S}_{\text {I }}$ profiles that were not well predicted by the model were those with high variability due to rapid fluctuations in patient condition, most likely due to changes in insulin and glucose secretion. This case is more serious as exogenous insulin is required, and a rapid increase in insulin sensitivity can lead to a decrease in BG levels. Such patients highlight the need for well designed insulin dosing protocols with safety checks. Currently, in addition to forward prediction of likely BG outcomes, STAR applies a maximum limit on the amount by which insulin can increase from treatment to treatment. This limit is dependent on BG and protects highly variable patients from excessive insulin therapy. 
There was a decrease in average percentage time in band and shift in distribution to around $90 \%$ when using $S_{I}$ only from periods where a patient had received insulin. The new stochastic bands are tighter and therefore less conservative, but better reflect patient condition and variability for patients actually receiving insulin, as shown in the improved coverage of the target band (4.0-8.0 $\mathrm{mmol} / \mathrm{L})$ and lower number of patients with hypoglycaemic events. It also suggests that the 7 patients with percentage time in band less than $60 \%$ have higher insulin sensitivity, and thus, as seen in the data, received less insulin therapy. In addition, uncertainty around the endogenous glucose and insulin secretion is highlighted, particularly in the patient recovery stage. This result gives direction for future work in better quantification of variability around endogenous secretion of insulin and glucose. It also underlines the models specificity for use in patients requiring insulin therapy. 


\subsection{CONCLUSIONS}

Stochastic model based forecasting based on a larger patient database provides a more accurate representation of the NICU population, but is limited by inter-patient variability. Birth weight and gestational age dependent stochastic forecasting can be used to further increase per-patient accuracy and coverage. The use of stochastic models based on insulin sensitivities from insulin treatment periods better reflected patient condition in control, as reflected by the $10 \%$ increase in percentage time in band and the halving of the number of patients with hypoglycaemic events. Further improvement can be achieved through investigations into the variability associated with endogenous insulin and glucose production.

\subsection{REFERENCES}

Agus, M. S., Javid, P. J., Ryan, D. P., et al. (2004). Intravenous insulin decreases protein breakdown in infants on extracorporeal membrane oxygenation. Journal of Pediatric Surgery, 39(6), 839-844.

Alaedeen, D. I., Walsh, M. C., \& Chwals, W. J. (2006). Total parenteral nutrition-associated hyperglycemia correlates with prolonged mechanical ventilation and hospital stay in septic infants. Journal of Pediatric Surgery, 41(1), 239-244; discussion 239-244. doi: 10.1016/j.jpedsurg.2005.10.045

Alsweiler, J. M., Kuschel, C. A., \& Bloomfield, F. H. (2007). Survey of the management of neonatal hyperglycaemia in Australasia. Journal of Paediatrics and Child Health, 43(9), 632-635.

Beardsall, K., Ogilvy-Stuart, A. L., Frystyk, J., et al. (2007). Early elective insulin therapy can reduce hyperglycemia and increase insulin-like growth factor-I levels in very low birth weight infants. The Journal of pediatrics, 151(6), 611-617.

Beardsall, K., Vanhaesebrouck, S., Ogilvy-Stuart, A. L., et al. (2007). A randomised controlled trial of early insulin therapy in very low birth weight infants, "NIRTURE" (neonatal insulin replacement therapy in Europe). BMC Pediatrics, 7, 29. doi: $10.1186 / 1471-2431-7-29$

Beardsall, K., Vanhaesebrouck, S., Ogilvy-Stuart, A. L., et al. (2010). Prevalence and determinants of hyperglycemia in very low birth weight infants: cohort analyses of the NIRTURE study. Journal of Pediatrics, 157(5), 715-719 e711-713. doi: 10.1016/j.jpeds.2010.04.032

Beardsall, K., Vanhaesebrouck, S., Ogilvy-Stuart, A. L., et al. (2008). Early Insulin Therapy in Very-Low-Birth-Weight Infants. The New England Journal of Medicine, 359(18), 1873-1884. doi: 10.1056/NEJMoa0803725

Chase, J. G., Le Compte, A. J., Suhaimi, F., et al. (2011). Tight glycemic control in critical care - The leading role of insulin sensitivity and patient variability: A review and 
model-based analysis. Computer Methods and Programs in Biomedicine, 102(2), 156171. doi: 10.1016/j.cmpb.2010.11.006

Chase, J. G., Suhaimi, F., Penning, S., et al. (2010). Validation of a model-based virtual trials method for tight glycemic control in intensive care. Biomedical Engineering Online, 9, 84. doi: 10.1186/1475-925X-9-84

Cowett, R. M., \& Farrag, H. M. (2004). Selected principles of perinatal-neonatal glucose metabolism. Seminars in Neonatology, 9(1), 37-47.

Dickson, J. L., Le Compte, A. J., Floyd, R. P., et al. Development and optimisation of stochastic targeted (STAR) glycaemic control for pre-term infants in neonatal intensive care. Biomedical Signal Processing and Control(0). doi: 10.1016/j.bspc.2012.08.002

Hann, C., Chase, J., \& Shaw, G. (2006). Integral-based Identification of Patient Specific Parameters for a Minimal Cardiac Model. Computer Methods and Programs in Biomedicine, 81(2), 181-192.

Hays, S. P., Smith, B., \& Sunehag, A. L. (2006). Hyperglycemia Is a Risk Factor for Early Death and Morbidity in Extremely Low Birth-Weight Infants. Pediatrics, 118(5), 1811-1818.

Heimann, K., Peschgens, T., Kwiecien, R., et al. (2007). Are recurrent hyperglycemic episodes and median blood glucose level a prognostic factor for increased morbidity and mortality in premature infants $</=1500 \mathrm{~g}$ ? Journal of Perinatal Medicine, 35(3), 245-248.

Hemachandra, A. H., \& Cowett, R. M. (1999). Neonatal Hyperglycemia. Pediatrics in Review, 20(7), 16e-24.

Le Compte, A. J., Chase, J. G., Lynn, A., et al. (2009). Blood Glucose Controller for Neonatal Intensive Care: Virtual trials development and 1st clinical trials. Journal of Diabetes Science and Technology, 3(5), 1066-1081.

Le Compte, A. J., Lee, D. S., Chase, J. G., et al. (2010). Blood glucose prediction using stochastic modeling in neonatal intensive care. IEEE Transactions on Biomedical Engineering, 57(3), 509-518. doi: 10.1109/TBME.2009.2035517

Le Compte, A. J., Lynn, A. M., Lin, J., et al. (2012). Pilot study of a model-based approach to blood glucose control in very-low-birthweight neonates. BMC Pediatrics, 12(1), 117. doi: $10.1186 / 1471-2431-12-117$

Lin, J., Lee, D., Chase, J., et al. (2006). Stochastic Modelling of Insulin Sensitivity Variability in Critical Care. Biomedical Signal Processing \& Control, 1, 229-242.

Meetze, W., Bowsher, R., Compton, J., et al. (1998). Hyperglycemia in extremely- low-birthweight infants. Biology of the Neonate, 74(3), 214-221. 


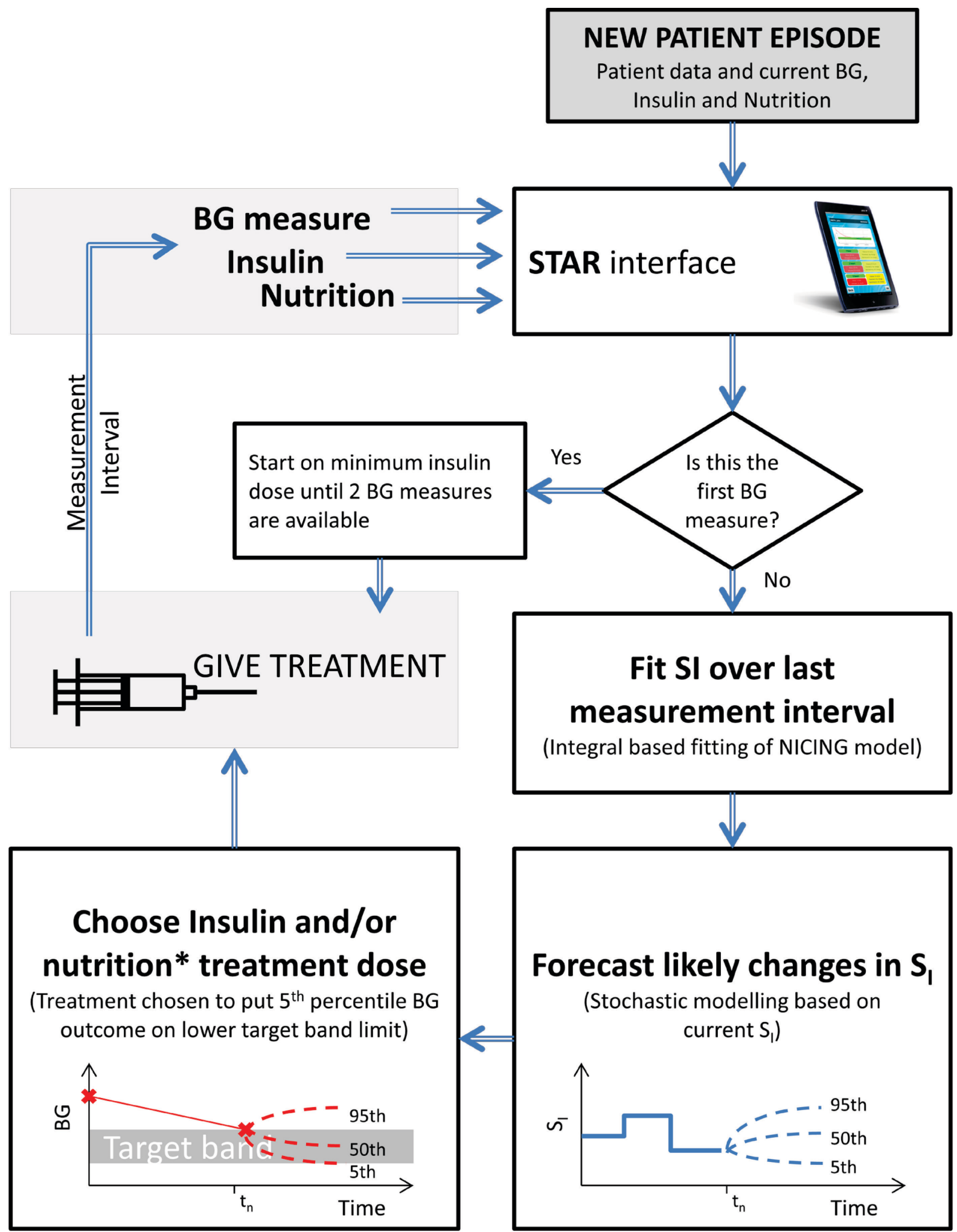

*Currently nutrition is clinically modulated. In the adult ICU STAR modulates both nutrition and insulin to optimise glycaemic control. 
APPENDIX 2: Virtual trial simulation algorithm

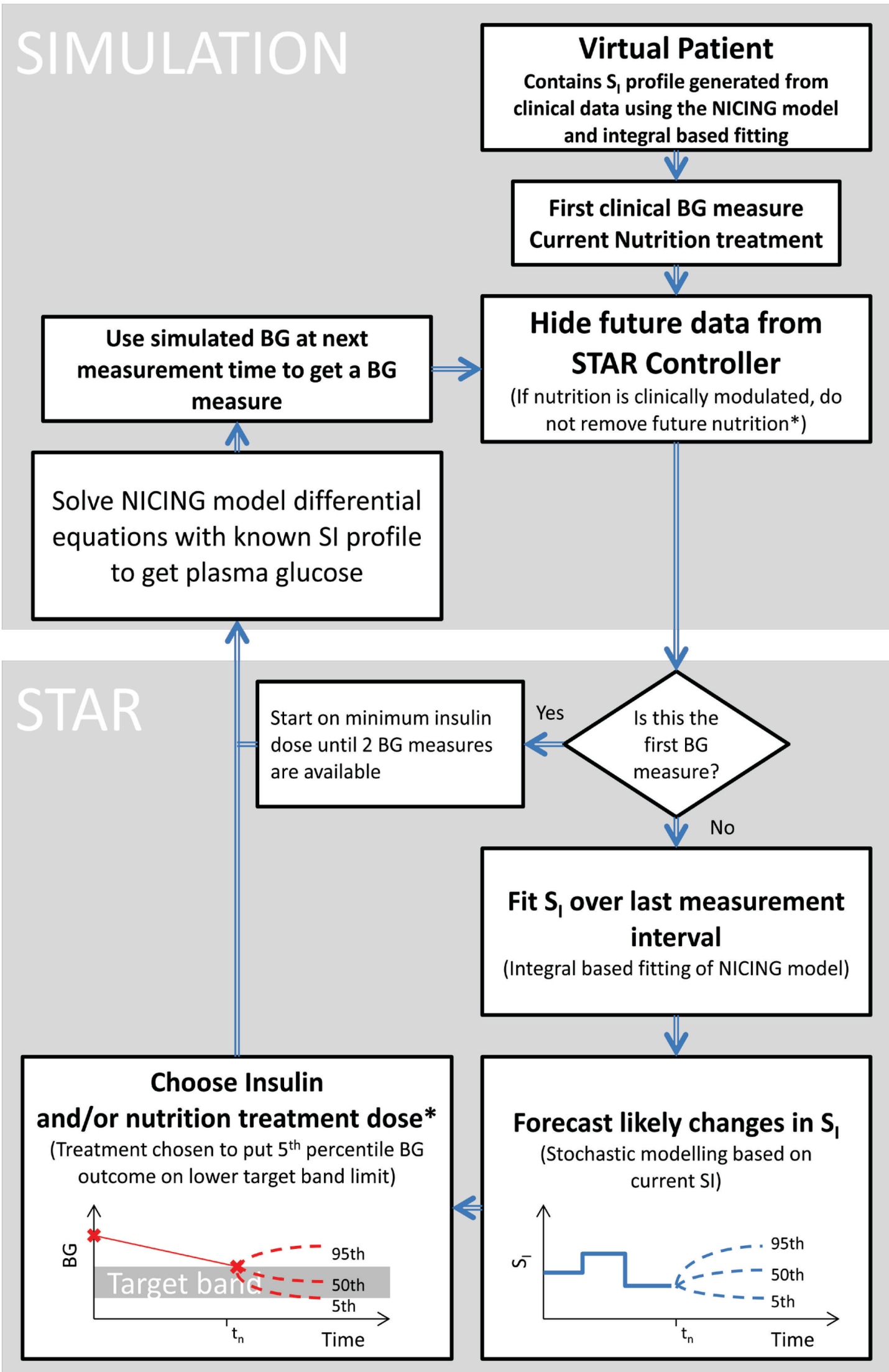

*Currently nutrition is clinically modulated. In the adult ICU STAR modulates both nutrition and insulin to optimise glycaemic control. 